\title{
Control of Production-Distribution Systems under Discrete Disturbances and Control Actions
}

\author{
Dario Bauso and Danielle C. Tarraf
}

\begin{abstract}
This paper deals with the robust control and optimization of production-distribution systems. The model used in our problem formulation is a general network flow model that describes production, logistics, and transportation applications. The novelty in our formulation is in the discrete nature of the control and disturbance inputs. We highlight three main contributions: First, we derive a necessary and sufficient condition for the existence of robustly control invariant hyperboxes. Second, we show that a stricter version of the same condition is sufficient for global convergence to an invariant set. Third, for the scalar case, we show that these results parallel existing results in the setting where the control actions and disturbances are analog. We conclude with two simple illustrative examples.
\end{abstract}

\section{INTRODUCTION}

By production-distribution systems we mean any system where resources at geographically distributed warehouses are converted into products with (in the case of production) or without (in the case of distribution) physical modifications of the goods, commodities, materials involved in the process. In this sense, the term is quite general, encompassing both production and logistics models.

Production-distribution systems can be thought of as network flow problems, as they are efficiently described using graphs or hypergraphs with nodes and arcs associated with resources/products and flows, respectively. The accumulated discrepancy over time of the input and output flows at $n$ interconnected nodes is captured by the $n$-dimensional state of the system: Practically, this represents resources and or products available (stored) at the $n$ warehouses. The control input denotes the controlled flows (i.e. the production and/or distribution) and the disturbance input denotes the uncontrolled flows (i.e. the demand). The dynamics of the system are thus linear, with the matrices defining the model describing which and in what quantity resources and products are involved in a unit flow.

Practically, resource/product inventory stored at the warehouses translate into holding costs for the warehouse manager, and so one desires to keep the inventory bounded and as close as possible to the unknown demand. On the other hand, keeping the inventory close to zero may induce shortages and subsequent loss of clients for the warehouse manager, hence there is a tradeoff. Questions of existence of robustly control invariant sets, as well as their properties when they do

D. Bauso is with Dipartimento di Ingegneria Chimica, Gestionale, Informatica e Meccanica, Università di Palermo, Italy (dario.bauso@unipa.it).

D. C. Tarraf is with the Department of Electrical and Computer Engineering, Johns Hopkins University, Baltimore, MD 21218, USA (dtarrafejhu.edu). exist, are thus of interest. Of course, this problem has been previously considered in the literature, for instance in [6] [7] where polytopic invariant sets as considered and in [5] where ellipsoidal sets are considered. Restricting the discussion to polytopic sets has the advantage of reducing the problem to linear programs, with the relative computational benefits: The problem turns into a robust quadratic programming problem involving linear matrix inequalities when the sets are ellipsoidal.

The novelty of this paper is in the discrete nature of the inputs, justifiable from practical as well as theoretical perspectives. From a practical standpoint, materials and goods are usually processed in batches. From a theoretical standpoint, the study of systems under discrete controls and disturbances has sparked much interest in recent years as evidenced by the literature on alphabet control [8] [9], [10], mixed integer model predictive control [1], discrete team theory [11] and boolean control [3]. In general, the problems of interest may be formulated as min-max games [2].

Our contribution consists of three main results. First, we derive a necessary and sufficient condition for the existence of robustly control invariant hyperboxes. Second, we show that a stricter version of the above condition is sufficient to guarantee global convergence of all state trajectories to the invariant set. Finally, we show that these two results when specialized to the scalar case share striking similarities to existing results in the literature where the inputs are assumed to be analog [6] [7].

The paper is organized as follows: We formulate the problem in Section II. We state and prove the main results in Section III, and we discuss their specialization for the scalar case in the context of existing work. We present two simple illustrative examples in Section IV and conclude in Section V.

A word on notation: $\mathbb{R}$ and $\mathbb{Z}$ denote the set of reals and integers, respectively. $[x]_{i}$ denotes the $i^{\text {th }}$ component of vector $x \in \mathbb{R}^{n}$. hull $\{S\}$ and $\operatorname{int}(S)$ denote the convex hull and interior, respectively, of set $S \subset \mathbb{R}^{n} . \mathbb{B}^{n}$ denotes the set of vertices of the unit hypercube, that is $\mathbb{B}^{n}=\{0,1\}^{n}$.

\section{SETUP OF THE PROBLEM}

\section{A. Problem Statement}

Consider the system described by

$$
x(t+1)=x(t)+B u(t)-D w(t)
$$

where time index $t \in \mathbb{Z}_{+}$, state $x(t) \in \mathbb{R}^{n}$, control input $u(t) \in \mathcal{U}^{m}$ and disturbance input $w(t) \in \mathcal{W}^{p}$. The control alphabet set $\mathcal{U}=\left\{a_{1}, \ldots, a_{r}\right\}$ is a discrete, ordered set with 
$a_{1}<\ldots<a_{r}$. Likewise the disturbance alphabet set $\mathcal{W}=$ $\left\{b_{1}, \ldots, b_{q}\right\}$ is a discrete ordered set with $b_{1}<\ldots<b_{q}$. Matrices $B \in\{-1,0,1\}^{n \times m}$ and $D \in\{-1,0,1\}^{n \times p}$ are given.

Definition 1: A set $X=\left[0, x_{1}^{+}\right] \times \ldots \times\left[0, x_{n}^{+}\right] \subset \mathbb{R}^{n}$, $x_{i}^{+}>0$, is robustly control invariant if there exists a control law $\varphi: X \rightarrow \mathcal{U}^{m}$ such that for every $x(t) \in X, x(t+$ $1)=x(t)+B \varphi(x(t))-D w(t) \in X$ for any disturbance $w(t) \in \mathcal{W}^{p}$.

Remark 1: When $X=\left[0, x_{1}^{+}\right] \times \ldots \times\left[0, x_{n}^{+}\right]$is robustly control invariant, then so is any other hyperbox $X^{\prime}=$ $\left[x_{1}^{-}, x_{1}^{-}+x_{1}^{+}\right] \times \ldots \times\left[x_{n}^{-}, x_{n}^{-}+x_{n}^{+}\right]$. Indeed, control law $\varphi^{\prime}: X^{\prime} \rightarrow \mathcal{U}^{m}$ defined by $\varphi^{\prime}(x)=\varphi\left(x-x^{-}\right)$, where $\left[x^{-}\right]_{i}=x_{i}^{-}$, verifies this assertion.

Definition 2: A robustly control invariant set $X=$ $\left[0, x_{1}^{+}\right] \times \ldots \times\left[0, x_{n}^{+}\right] \subset \mathbb{R}^{n}$ is globally attractive if there exists a control law $\psi: \mathbb{R}^{n} \backslash X \rightarrow \mathcal{U}^{m}$ such that for every initial condition $x(0) \in \mathbb{R}^{n} \backslash X$ and disturbance $w: \mathbb{Z}_{+} \rightarrow \mathcal{W}^{p}$, there exists a $\tau \in \mathbb{Z}_{+}$such that $x(\tau) \in X$.

We are interested in answering two questions for this class of systems:

Question 1: Under what conditions does a robustly control invariant set $X$ exist?

Question 2: Under what conditions is a robustly control invariant set $X$ globally attractive?

\section{B. Significance of the Problem Statement}

Dynamics (1) describes the evolution of productiondistribution systems where the state $x(t)$ represents resources and or products available (stored) at the $n$ warehouses, the control input $u(t)$ denotes the controlled (productiondistribution) flows and the disturbance input $w(t)$ the uncontrolled flows. Matrix $B$ establishes which and in what quantity resources and products are involved in a unit flow. Observe that depending on the sign of the $i j$ th entry in $B$ the same $x_{i}$ is a resource (negative sign) or a product (positive sign) for flow $j$.

An efficient way to describe production-distribution systems is by using hypergraphs with nodes and arcs associated to resources/products and flows respectively. For the example in Fig. 1 borrowed from [7], we have three resourses/products $x_{i}, i=1,2,3$, four controlled flows $u_{i}, i=1, \ldots, 4$ and five uncontrolled flows $w_{i}, i=1, \ldots, 5$. One unit of flow $u_{1}$ produces one unit of product A associated to $x_{1}$. Same for flow $u_{2}$ and product $B$ associated to $x_{2}$. One unit of flow $u_{3}$ uses one unit of resources $\mathrm{A}$ and $\mathrm{B}$ to produce one unit of product $\mathrm{AB}$ associated to $x_{3}$. One unit of flow $u_{4}$ uses one unit of resources A to produce one unit of product B. Similar explanation for the uncontrolled flows with the only exception that these are exogenously set. So, one unit of flow $w_{1}$ is a unitary demand of resource $\mathrm{A}$, and similarly for $w_{2}$ and $w_{3}$ with resource $\mathrm{B}$ and $\mathrm{AB}$ respectively. Flow $w_{4}$ uses
A to produce $\mathrm{AB}$ as well as $w_{5}$ uses $\mathrm{B}$ to produce $\mathrm{AB}$. The highlighted relations between flows and resources/products are described by matrices

$$
\begin{aligned}
B= & {\left[\begin{array}{rrrr}
1 & 0 & -1 & -1 \\
0 & 1 & -1 & 1 \\
0 & 0 & 1 & 0
\end{array}\right] } \\
D= & {\left[\begin{array}{rrrrr}
-1 & 0 & 0 & -1 & 0 \\
0 & -1 & 0 & 0 & -1 \\
0 & 0 & -1 & 1 & 1
\end{array}\right] . }
\end{aligned}
$$

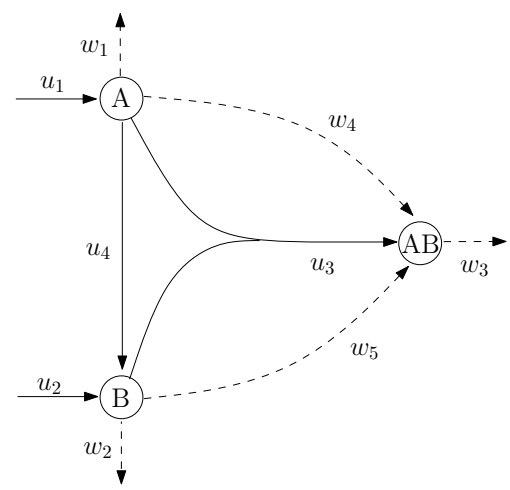

Fig. 1. Network system with three resourses/products, four controlled (solid) and five uncontrolled (dashed) flows.

From the example, the motivation of looking at the smallest robustly controlled invariant set is clear. Since resource/product inventory stored at the warehouse translate into holding costs for the warehouse manager, we may want to keep the inventory bounded and as close as possible to the expected demand (uncontrolled flows), this one being unknown. At the same time, keeping the inventory close to zero may induce shortages (lost of clients) for the warehouse manager. One practical way to deal with robustly controlled invariant set is to apriori impose some structure and restrict the search to polytopic or ellipsoidal sets (cfg. [4], [5], [6], [7]). The simplicity with which these sets can be formulated translates into computational tractability for the optimization problems involved in the study (linear or quadratic mathematical programs).

\section{MAIN RESULTS}

Consider the following sets for $i \in\{1, \ldots, n\}$

$$
\begin{aligned}
\mathcal{U}_{+}^{i} & =\left\{u \in \mathcal{U}^{m} \mid[B u-D w]_{i} \geq 0, \forall w \in \mathcal{W}^{p}\right\} \\
\mathcal{U}_{-}^{i} & =\left\{u \in \mathcal{U}^{m} \mid[B u-D w]_{i} \leq 0, \forall w \in \mathcal{W}^{p}\right\} \\
\mathcal{U}_{+*}^{i} & =\left\{u \in \mathcal{U}^{m} \mid[B u-D w]_{i}>0, \forall w \in \mathcal{W}^{p}\right\} \\
\mathcal{U}_{-*}^{i} & =\left\{u \in \mathcal{U}^{m} \mid[B u-D w]_{i}<0, \forall w \in \mathcal{W}^{p}\right\},
\end{aligned}
$$

associate with every $x \in \mathbb{R}_{+}^{n}$ a signature, namely an n-tuple $\left(s_{1}, \ldots, s_{n}\right)$ with $s_{i}=+$ if $[x]_{i}=0$ and $s_{i}=-$ if $[x]_{i}>0$, and two subsets of $\mathcal{U}^{m}$ defined by

$$
\begin{gathered}
\mathcal{U}_{x}=\mathcal{U}_{s_{1}}^{1} \cap \ldots \cap \mathcal{U}_{s_{n}}^{n}, \\
\mathcal{U}_{x}^{*}=\mathcal{U}_{s_{1} *}^{1} \cap \ldots \cap \mathcal{U}_{s_{n} *}^{n} .
\end{gathered}
$$

We are now ready to present the main results. 


\section{A. Existence of a Robustly Control Invariant Set}

Theorem 1: The following two statements are equivalent:

(a) There exists a set $X=\left[0, x_{1}^{+}\right] \times \ldots \times\left[0, x_{n}^{+}\right]$that is robustly control invariant.

(b) The condition

$$
\mathcal{U}_{z} \neq \emptyset
$$

holds for all $z \in \mathbb{B}^{n}$.

Proof: (a) $\Rightarrow$ (b): Assume that $\mathcal{U}_{z}=\emptyset$ for some $z \in \mathbb{B}^{n}$ with signature $\left(s_{1}, \ldots, s_{2}\right)$. Consider a set $X=\left[0, x_{1}^{+}\right] \times$ $\ldots \times\left[0, x_{n}^{+}\right], x_{i}^{+}>0$, and a $u \in \mathcal{U}^{m}$. By assumption, there exists an $i \in\{1, \ldots, n\}$ such that $u \notin \mathcal{U}_{s_{i}}^{i}$. Now pick the vertex $x$ of $X$ given by

$$
[x]_{j}=\left\{\begin{array}{cc}
0 & s_{j}=+ \\
x_{j}^{+} & s_{j}=-
\end{array}\right.
$$

Note that the signatures of $z$ and $x$ are identical by construction. Letting $x(t)=x$ and applying control input $u(t)=u$, we have

$$
[x(t+1)]_{i}=[x(t)+B u(t)-D w(t)]_{i}=[x]_{i}+[B u-D w]_{i},
$$

and $[x(t+1)]_{i}-[x(t)]_{i}=[B u(t)-D w(t)]_{i}$ satisfies

$$
[x(t+1)]_{i}-[x(t)]_{i}>0
$$

for some $w \in \mathcal{W}^{p}$ when $[x]_{i} \neq 0$ and

$$
[x(t+1)]_{i}-[x(t)]_{i}<0
$$

for some $w \in \mathcal{W}^{p}$ when $[x]_{i}=0$. Hence $x(t+1) \notin X$ for some $w \in \mathcal{W}^{p}$. Noting that the choice of $u$ was arbitrary allows us to conclude that $X$ is not robustly control invariant. Noting that the choice of $X$ was arbitrary allows us to conclude that a robustly control invariant set cannot exist, thus completing our argument.

(b) $\Rightarrow$ (a): The proof is constructive. Assume that $\mathcal{U}_{z} \neq \emptyset$ holds for all $z \in \mathbb{B}^{n}$, and pick $u_{z} \in \mathcal{U}_{z}$. Define

$$
L_{i}^{*}=\max _{w, z}\left|\left[B u_{z}-D w\right]_{i}\right|
$$

The set $X=\left[0,2 L_{1}^{*}\right] \times \ldots \times\left[0,2 L_{n}^{*}\right]$ is robustly control invariant. Indeed, consider the control law $\varphi: X \rightarrow \mathcal{U}^{m}$ defined by $\varphi(x)=u_{z(x)}$, where $z(x) \in \mathbb{B}^{n}$ is the unique vertex of the unit hypercube with signature $s_{i}=+$ if $[x]_{i} \leq$ $L_{i}^{*}$ and $s_{i}=-$ otherwise. Note that under this control law, we have for any $i \in\{1, \ldots, n\}$

$$
[x(t+1)]_{i}=[x(t)]_{i}+[B \varphi(x(t))-D w(t)]_{i}
$$

Thus by construction, when $0 \leq[x(t)]_{i} \leq L_{i}^{*}, 0 \leq$ $[B \varphi(x(t))-D w(t)]_{i} \leq L_{i}^{*}$ and $0 \leq[x(t+1)]_{i} \leq 2 L_{i}^{*}$. Likewise when $L_{i}<[x(t)]_{i} \leq 2 L_{i}^{*},-L_{i}^{*} \leq[B \varphi(x(t))-$ $D w(t)]_{i} \leq 0$ and $0 \leq[x(t+1)]_{i} \leq 2 L_{i}^{*}$. It follows that $X$ is robustly control invariant.

\section{B. Global Attractiveness}

Theorem 2: If condition

$$
\mathcal{U}_{z}^{*} \neq \emptyset
$$

holds for all $z \in \mathbb{B}^{n}$, there exists a set $X=\left[0, x_{1}^{+}\right] \times$ $\ldots \times\left[0, x_{n}^{+}\right]$that is robustly control invariant and globally attractive.

Proof: The proof is constructive. For each $z \in \mathbb{B}^{n}$, pick $u_{z} \in \mathcal{U}_{z}^{*}$. Define

$$
L_{i}^{*}=\max _{w, z}\left|[B u(z)-D w]_{i}\right|
$$

The set $X=\left[0,2 L_{1}^{*}\right] \times \ldots \times\left[0,2 L_{n}^{*}\right]$ is robustly control invariant and globally attractive. Indeed, consider the control law $\zeta: \mathbb{R}^{n} \rightarrow \mathcal{U}^{m}$ defined by $\zeta(x)=u_{z(x)}$, where $z(x) \in$ $\mathbb{B}^{n}$ is the unique vertex of the unit hypercube with signature $s_{i}=+$ if $[x]_{i} \leq L_{i}^{*}$ and $s_{i}=-$ otherwise. Noting that $\mathcal{U}_{z}^{*} \subseteq \mathcal{U}_{z}$, it immediately follows that $X$ is robustly control invariant by a proof identical to the proof of sufficiency in Theorem 1. To prove global attractiveness, let

$$
\Delta=\min _{i} \min _{z, w}\left|\left[B u_{z}-D w\right]_{i}\right|
$$

and consider the function $V: \mathbb{R}^{n} \rightarrow \mathbb{R}$ defined by

$$
V(x)=\max _{i} \min _{y \in X}\left|[x]_{i}-[y]_{i}\right| .
$$

Note that by construction, we have:

- $\Delta>0$.

- $V(x) \geq 0$, for all $x \in \mathbb{R}^{n}$.

- $V(x)=0 \Leftrightarrow x \in X$.

When control law $\zeta$ is utilized, we can show that along system trajectories, we have

$$
V(x(t+1))-V(x(t))<0
$$

whenever $x(t) \in \mathbb{R}^{n} \backslash X$. The details are omitted here, but the idea is to appropriately partition the state-space into polytopes in which this inequality can be verified. Moreover, we have

$$
V(x(t+1)) \leq V(x(t))-\Delta
$$

whenever $x(t) \in \mathbb{R}^{n} \backslash X$ and $x(t+1) \in \mathbb{R}^{n} \backslash X$.

Thus, for any choice of initial condition $x(0)$ and of disturbance input $w: \mathbb{Z}_{+} \rightarrow \mathcal{W}^{p}$, we conclude from (4) that $\lim _{t \rightarrow \infty} V(x(t)) \rightarrow 0$. Moreover, we conclude from (5) that there must exist a $\tau>0$ such that $V(x(\tau))=0$, or equivalently $x(\tau) \in X$. Hence $X$ is indeed globally attractive.

\section{Interpretation of the Main Results in the Scalar Case}

In this section, we consider the special case of scalar dynamics in order to point out that Theorem 1 is strikingly parallel to existing results for the setup where the control and disturbance are assumed to take continuous values.

Consider the dynamics described by

$$
x(t+1)=x(t)+\alpha u(t)-\eta w(t)
$$


where $\alpha$ and $\eta$ are given non-zero scalars, $u(t) \in \mathcal{U}$ and $w(t) \in \mathcal{W}$.

The relevant sets can be computed by looking at the signs of the entries of the following table

\begin{tabular}{l||c|c|c}
$u / w$ & $b_{1}$ & $\ldots$ & $b_{q}$ \\
\hline \hline$a_{1}$ & $\alpha a_{1}-\eta b_{1}$ & $\ldots$ & $\alpha a_{1}-\eta b_{q}$ \\
\hline$\vdots$ & $\vdots$ & & $\vdots$ \\
\hline$a_{r}$ & & &
\end{tabular}

Specifically, we have the following subsets of $\mathcal{U}$ :

$$
\begin{gathered}
\mathcal{U}_{+}=\{u \in \mathcal{U} \mid \alpha u-\eta w \geq 0, \forall w \in \mathcal{W}\} \\
\mathcal{U}_{-}=\{u \in \mathcal{U} \mid \alpha u-\eta w \leq 0, \forall w \in \mathcal{W}\} \\
\mathcal{U}_{+*}=\{u \in \mathcal{U} \mid \alpha u-\eta w>0, \forall w \in \mathcal{W}\} \\
\mathcal{U}_{-*}=\{u \in \mathcal{U} \mid \alpha u-\eta w<0, \forall w \in \mathcal{W}\}
\end{gathered}
$$

Note that $\mathcal{U}_{+*} \subseteq \mathcal{U}_{+}$and $\mathcal{U}_{-*} \subseteq \mathcal{U}_{-}$.

Lemma 1: $\mathcal{U}_{+} \neq \emptyset$ and $\mathcal{U}_{-} \neq \emptyset$ iff $h u l l\{\eta \mathcal{W}\} \subseteq$ hull $\{\alpha \mathcal{U}\}$.

Proof: To prove sufficiency, assume that one of the two sets, say $\mathcal{U}_{+}$, is empty. Thus for every $u \in \mathcal{U}$, there exists a $w \in \mathcal{W}$ such that $\alpha u-\eta w<0$. In particular, there exists $w \in \mathcal{W}$ such that $\alpha a_{m}-\eta w<0$, equivalently $\eta w>\alpha a_{m}$, and hence there exists an element $y \in \operatorname{hull}\{\eta \mathcal{W}\}$ such that $y \notin \operatorname{hull}\{\alpha \mathcal{U}\}$.

To prove necessity, assume that $\mathcal{U}_{+} \neq \emptyset$ and $\mathcal{U}_{-} \neq \emptyset$. Thus there exists $u_{1} \in \mathcal{U}_{+}$and $u_{2} \in \mathcal{U}_{-}$satisfying

$$
\left\{\begin{array}{l}
\alpha u_{1}-\eta w \geq 0 \\
\alpha u_{2}-\eta w \leq 0
\end{array}\right.
$$

for all $w \in \mathcal{W}$. Since $a_{m} \geq u_{1}$ and $a_{1} \leq u_{2}$, we have

$$
\left\{\begin{array} { c } 
{ \alpha a _ { m } - \eta w \geq 0 } \\
{ \alpha a _ { 1 } - \eta w \leq 0 }
\end{array} \Leftrightarrow \left\{\begin{array}{c}
\alpha a_{m} \geq \eta w \\
\alpha a_{1} \leq \eta w
\end{array}\right.\right.
$$

for all $w \in \mathcal{W}$. In particular, it holds that $\alpha a_{m} \geq \eta b_{p}$ and $\alpha a_{1} \leq \eta b_{1}$ hence hull $\{\eta \mathcal{W}\} \subseteq \operatorname{hull}\{\alpha \mathcal{U}\}$.

Corollary 1: The following two statements are equivalent:

1) There exists a finite set $X=\left[0, x^{+}\right]$that is robustly control invariant.

2) $\operatorname{hull}\{\eta \mathcal{W}\} \subseteq \operatorname{hull}\{\alpha \mathcal{U}\}$.

Proof: Noting that condition (2) is equivalent to the condition $\mathcal{U}_{+} \neq \emptyset$ and $\mathcal{U}_{-} \neq \emptyset$ in the scalar setting, the statement of the corollary follows from Lemma 1 and Theorem 1.

Remark 2: The statement of Corollary 1 reminds us of the well known set dominance conditions provided in [7] for the discrete-time flow model with continuous inputs. The use of the hull(.) operator is made necessary as in our case the alphabet sets $\mathcal{U}$ and $\mathcal{W}$ are discrete sets rather than polytopes as in [7]. Similar conditions have been shown to play the same important role also in [6] for the continuoustime version: Future work will investigate the relevance of these conditions for the case of continuous time system with discrete control and disturbance alphabets
Lemma 2: Suppose $\mathcal{U}_{+} \neq \emptyset$ and $\mathcal{U}_{-} \neq \emptyset$. We have

$\mathcal{U}_{+*} \neq \emptyset$ or $\mathcal{U}_{-*} \neq \emptyset \Leftrightarrow \operatorname{hull}\{\eta \mathcal{W}\} \subset \operatorname{hull}\{\alpha \mathcal{U}\}$.

Proof: To prove sufficiency, assume that hull $\{\eta \mathcal{W}\} \subset$ $\operatorname{hull}\{\alpha \mathcal{U}\}$. Thus there exists at least one element $x$ in $\operatorname{hull}\{\alpha \mathcal{U}\}$ and not in hull $\{\eta \mathcal{W}\}$. If $x$ is strictly larger than every element in $h u l l\{\eta \mathcal{W}\}$, then $\alpha a_{m}$ is also strictly larger than every element in $h u l l\{\eta \mathcal{W}\}, \alpha a_{m}-\eta w>0$ for all $w \in \mathcal{W}, a_{m} \in \mathcal{U}_{+*}$, and $\mathcal{U}_{+*} \neq \emptyset$. Otherwise, $x$ must be strictly smaller than every element in $\operatorname{hull}\{\eta \mathcal{W}\}$, and by a similar argument $\mathcal{U}_{-*} \neq \emptyset$.

To prove necessity, assume that $\mathcal{U}_{+*} \neq \emptyset$ or $\mathcal{U}_{-*} \neq \emptyset$. If there exists $u_{1} \in \mathcal{U}_{+*}$ satisfying $\alpha u_{1}-\eta w>0$ for all $w \in \mathcal{W}$, then since $a_{m} \geq u_{1}$ we have $\alpha a_{m}-\eta w>0 \Leftrightarrow$ $\alpha a_{m}>\eta w$ for all $w \in \mathcal{W}$. In particular it holds that $\alpha a_{m}>$ $\eta b_{p}$, hence $\operatorname{hull}\{\eta \mathcal{W}\} \subset \operatorname{hull}\{\alpha \mathcal{U}\}$. Otherwise there must exist a $u_{2} \in \mathcal{U}_{-*}$ satisfying $\alpha u_{2}-\eta w<0$ for all $w \in \mathcal{W}$. Since $a_{1} \leq u_{2}$, we have $\alpha a_{1}-\eta w<0 b \Leftrightarrow \alpha a_{1}<\eta w$, for all $w \in \mathcal{W}$. In particular it holds $\alpha a_{1}<\eta b_{1}$ and hence $\operatorname{hull}\{\eta \mathcal{W}\} \subset \operatorname{hull}\{\alpha \mathcal{U}\}$.

\section{ILLUSTRATIVE EXAMPLES}

\section{A. A Scalar System}

Let us start with a scalar example. Consider dynamics (6) and take inputs $u \in U=\{-100,-2,3,150\}, w \in W=$ $\{-6,4\}$, and parameters $\alpha=\eta=1$. We first compute the relevant sets by direct inspection of the table below:

\begin{tabular}{c||c|c|}
$u / w$ & -6 & 4 \\
\hline \hline-100 & -94 & -104 \\
\hline-2 & 4 & -6 \\
\hline 3 & 9 & -1 \\
\hline 150 & 156 & 146
\end{tabular}

We have $U_{+}=U_{+*}=\{150\}$ and $U_{-}=U_{-*}=\{-100\}$. Invoking Theorems 1 and 2, we conclude that a robustly control invariant set exists, and moreover it is globally attractive. Indeed, it is easy to verify that the set $X=[0,157]$ is one such set (in fact, the smallest!).

\section{B. Network with 2 Nodes}

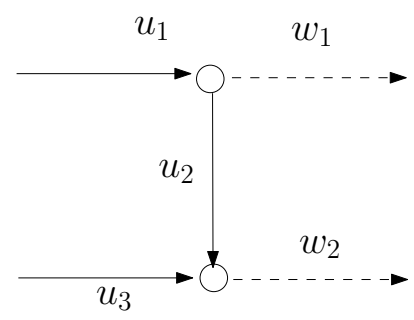

Fig. 2. Network system.

Consider the system depicted in Fig. 2 representing two warehouses, three controlled flows and two uncontrolled flows. A unit of flow $u_{1}$ produces one unit of product $x_{1}$. Flow $u_{2}$ uses one unit of $x_{1}$ to produce one unit of $x_{2}$. A unit of flow $u_{3}$ produces one unit of product $x_{2}$. Uncontrolled 
flows $u_{1}$ and $u_{2}$ represent the exogenous demand of resource $x_{1}$ and $x_{2}$ respectively. The associated dynamics are then:

$$
\begin{aligned}
{\left[\begin{array}{l}
x_{1}(t+1) \\
x_{2}(t+1)
\end{array}\right]=\left[\begin{array}{l}
x_{1}(t) \\
x_{2}(t)
\end{array}\right]+} & {\left[\begin{array}{ccc}
1 & -1 & 0 \\
0 & 1 & 1
\end{array}\right]\left[\begin{array}{l}
u_{1}(t) \\
u_{2}(t) \\
u_{3}(t)
\end{array}\right] } \\
& -\left[\begin{array}{l}
w_{1}(t) \\
w_{2}(t)
\end{array}\right]
\end{aligned}
$$

Assume that flows can only be processed in batches with $\mathcal{U}=$ $\{-5,-2,1,6\}$, and the disturbances also appear in batches with $\mathcal{W}=\{-3,2\}$.

We begin by computing the relevant sets:

$$
\begin{aligned}
\mathcal{U}_{+}^{1}= & \{(-2,-5, \mathrm{x}),(1,-5, \mathrm{x}),(1,-2, \mathrm{x}),(6,-5, \mathrm{x}), \\
& (6,-2, \mathrm{x}),(-6,1, \mathrm{x})\} \\
\mathcal{U}_{-}^{1}= & \{(-5,-2, \mathrm{x}),(-5,1, \mathrm{x}),(-5,6, \mathrm{x}),(-2,1, \mathrm{x}), \\
& (-2,6, \mathrm{x}),(1,6, \mathrm{x})\} \\
\mathcal{U}_{+}^{2}= & \{(\mathrm{x},-2,6),(\mathrm{x}, 1,1),(\mathrm{x}, 1,6),(\mathrm{x}, 6,-2), \\
& (\mathrm{x}, 6,1),(\mathrm{x},-6,6)\} \\
\mathcal{U}_{-}^{2}= & \{(\mathrm{x},-5,-5),(\mathrm{x},-5,-2),(\mathrm{x},-5,1),(\mathrm{x},-2,-5), \\
& (\mathrm{x},-2,-2),(\mathrm{x}, 1,-5)\}
\end{aligned}
$$

Note that, for instance, we write $(-2,-5, \mathrm{x})$ to mean any control vector with $u_{1}=-2$ and $u_{2}=-5$. Note that condition (2) holds. Indeed,

$$
\begin{aligned}
& u^{1}=(1,-2,6) \in U_{+}^{1} \cap U_{+}^{2} \\
& u^{2}=(1,-2,-5) \in U_{+}^{1} \cap U_{-}^{2}, \\
& u^{3}=(-5,1,-5) \in U_{-}^{1} \cap U_{-}^{2}, \\
& u^{4}=(-5,1,6) \in U_{-}^{1} \cap U_{+}^{2} .
\end{aligned}
$$

Invoking Theorem 1, we look for a robustly controlled invariant set. To do this, following the proof of Theorem 1, we first need to calculate sets $L_{i}^{j}$ which take on the following values:

$$
\begin{array}{cl}
L_{1}^{1}=\max _{w}|1+2-w|=6, & L_{1}^{3}=\max _{w}|-5-1-w|=8 \\
L_{2}^{1}=\max _{w}|-2+6-w|=7, & L_{2}^{3}=\max _{w}|1-5-w|=6 \\
L_{1}^{2}=\max _{w}|1+2-w|=6, & L_{1}^{4}=\max _{w}|-5-1-w|=8 \\
L_{2}^{2}=\max _{w}|-2-5-w|=9, & L_{2}^{1}=\max _{w}|1+6-w|=10
\end{array}
$$

Then, we need to look for the maximal values $L_{i}^{*}$ which yield

$$
\begin{aligned}
& L_{1}^{*}=\max _{j}\left\{L_{1}^{j}\right\}_{j}=\max \{6,6,8,8\}=8 \\
& L_{2}^{*}=\max _{j}\left\{L_{2}^{j}\right\}_{j}=\max \{7,9,6,10\}=10 .
\end{aligned}
$$

We can then conclude (see Remark 1) that the following set $X$ is robustly control invariant:

$$
X=\left\{x \in \mathbb{R}^{2} \mid-8 \leq x_{1} \leq 8,-10 \leq x_{2} \leq 10\right\} .
$$

In Fig. 3, we plot the evolution of $x_{1}(t)$ and $x_{2}(t)$ for $t=1, \ldots, 100$ with initial state $x(0)=\left[\begin{array}{ll}30 & 30\end{array}\right]^{T}$. The plot shows that the state $x(t)$ converges to $X=\left\{x \in \mathbb{R}^{2} \mid-8 \leq\right.$ $\left.x_{1} \leq 8,-10 \leq x_{2} \leq 10\right\}$.
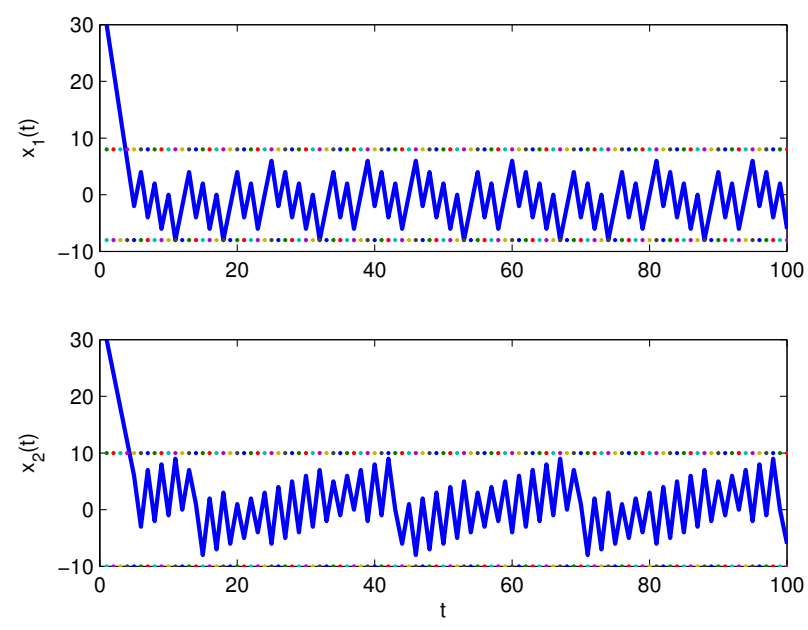

Fig. 3. Time plot of $x_{1}(t)$ and $x_{2}(t)$ for $t=1, \ldots, 100$ : in evidence the global convergence of $x(t)$ to $X=\left\{x \in \mathbb{R}^{2} \mid-8 \leq x_{1} \leq 8,-10 \leq\right.$ $\left.x_{2} \leq 10\right\}$.

\section{CONCLUSIONS AND FUTURE WORK}

In this paper we have provided a detailed analysis of existence conditions of robustly controlled invariant sets for production-distribution systems. The original aspect of the paper lies in the discrete nature of the control and disturbance inputs. Connections with existing results in the setup where the inputs are continuous are emphasized.

Our future research will focus on deriving bounds for the smallest invariant set, interpreting the derived necessary and sufficient conditions in the context of existing results for the general case, considering other models of discrete uncertainty, and revisiting the problem for continuous time 8 dynamics.

\section{ACKNOWLEDGMENTS}

D. C. Tarraf's research was supported by NSF CAREER 8 award ECCS 0954601 and AFOSR YIP award FA9550-11$1-0118$.

\section{REFERENCES}

[1] D. Axehill, L. Vandenberghe, and A. Hansson. Convex relaxations for mixed integer predictive control. Automatica, 46(5):1540-1545, June 2010.

[2] T. Basar and G.J. Olsder. Dynamic Noncooperative Game Theory. SIAM, 1999.

[3] D. Bauso. Boolean-controlled systems via receding horizon and linear programming. Journal of Mathematics of Control, Signals and Systems, 21(1):69-91, 2009.

[4] D. Bauso, F. Blanchini, and R. Pesenti. Optimization of long-run average-flow cost in networks with time-varying unknown demand. IEEE Trans. on Automatic Control, 55(1):20-31, 2010.

[5] D. Bauso, L. Giarrè, and R. Pesenti. Robust control of uncertain multiinventory systems via linear matrix inequality. International Journal of Control, 83(8):1723-1740, 2010. 
[6] F. Blanchini, S. Miani, and W. Ukovich. Control of productiondistribution systems with unknown inputs and system failures. IEEE Trans. on Automatic Control, 45(6):1072-1081, 2000.

[7] F. Blanchini, F. Rinaldi, and W. Ukovich. Least inventory control of multi-storage systems with non-stochastic unknown input. IEEE Trans. on Robotics and Automation, 13(5):633-645, 1997.

[8] G. C. Goodwin and D. E. Quevedo. Finite alphabet control and estimation. International Journal of Control, Automation and Systems, 1(4):412-430, 2003.

[9] D. C. Tarraf, A. Megretski, and M. A. Dahleh. Finite approximations of switched homogeneous systems for controller synthesis. IEEE Transactions on Automatic Control, 56(5):1140-1145, May 2011.

[10] D.C. Tarraf, A. Megretski, and M.A. Dahleh. A framework for robust stability for systems over finite alphabets. IEEE Transactions on Automatic Control, 53(5):1133-1146, June 2008.

[11] P. R. De Waal and J. H. Van Schuppen. A class of team problems with discrete action spaces: Optimality conditions based on multimodularity. SIAM Journal on Control and Optimization, 38(3):875-892, 2000. 